\title{
Substrate-Dependent Galvanotaxis of Directly Reprogrammed Human Neural Precursor Cells
}

\author{
Umalkhair Ahmed, MASc, ${ }^{1}$ Stephanie N. Iwasa, PhD, ${ }^{2}$ Laura Poloni, PhD, ${ }^{3}$ Jan-Eric Ahlfors, ${ }^{4}$ \\ Christopher Yip, PhD, ${ }^{1,3}$ Milos R. Popovic, PhD, ${ }^{1,5}$ and Cindi M. Morshead, PhD ${ }^{1,2,5}$
}

\begin{abstract}
Background: Neural precursor cells (NPCs) hold great promise for neural repair. Endogenous NPCs, found in the subventricular zone of the adult brain, proliferate and migrate toward lesion sites; however, it is not sufficient for neural repair. NPCs are electrosensitive cells that undergo directed migration in an electric field (EF). Here, we examined the EF-induced migration of a clinically relevant human NPC population.

Materials \& Methods: We examined the effects of different substrates and microenvironments on human NPC galvanotaxis.

Results: Human NPCs increased their migration speed in the presence of an EF, and the direction of migration (anodal vs. cathodal) varied between substrates. The secretome and extracellular $\mathrm{pH}$ were not significant factors in EF-induced migration; however, our results are consistent with substrate stiffness playing a role in the direction of cell migration.

Conclusion: These findings provide insight into the importance of the microenvironment on modulating human NPC migration and highlight substrate-dependent considerations for neurorepair.

Keywords: galvanotaxis, human neural precursor cells, microenvironment, cell migration, electrical stimulation
\end{abstract}

\section{Introduction}

A DULT NEURAL PRECURSOR cells (NPCs) show great promise for the repair of central nervous system (CNS) tissue after injury or disease. CNS injury can stimulate resident NPCs in the periventricular region of the adult brain to migrate to sites of injury and differentiate into mature neural phenotypes. However, the efficacy of this injury response is not sufficient to promote neural repair. ${ }^{1}$ One of the goals of regenerative medicine is to enhance this neural regenerative response of NPCs. NPCs are electrosensitive cells whereby applied electric fields (EFs) can promote rapid and directed murine NPC migration, a phenomenon known as galvanotaxis. ${ }^{2}$ Herein, we examined the galvanotactic response of human-derived NPCs with a view toward human application.

To develop the applied EFs as a neurorepair strategy and guide an increased number of NPCs to sites of injury in the brain, an understanding of the factors that influence human NPC galvanotaxis is needed. One unknown component of galvanotaxis is the underlying mechanisms that regulate the directedness of cell migration (cathodal vs. anodal). The di- rection can vary from cell type to cell type. Interestingly, cells derived from the same lineage can migrate in opposite directions in a similar range of field strengths $(0.1-1 \mathrm{~V} / \mathrm{mm})$. Osteoclasts migrate toward the positive electrode (anode), and osteoblasts migrate toward the negative electrode (cathode). ${ }^{3}$ Similarly, differential migration direction in the same population of cells (bovine epithelial cells) has been shown to be dependent on the strength of the EF. ${ }^{4}$ These findings highlight the importance of the cell type along with EF strength in understanding the galvanotactic response of distinct cell populations.

Extracellular matrix (ECM) components comprising the substrate can also influence cellular responses. Mechanical properties and composition of the ECM, including stiffness, have been found to regulate both short- and long-term cellular functions such as cell spreading, proliferation, and differentiation profile, ${ }^{5,6}$ in addition to integrin receptor expression, localization, and cytoskeletal organization. ${ }^{6}$ Further, Gilbert et al. found that the ECM can regulate tissue-specific stem cell behavior where the elastic modulus of a substrate can regulate muscle stem cell fate in culture. ${ }^{7}$ Muscle stem cells cultured

\footnotetext{
${ }^{1}$ Institute of Biomaterials and Biomedical Engineering, Departments of ${ }^{2}$ Surgery and ${ }^{3}$ Chemical Engineering and Applied Chemistry, University of Toronto, Toronto, Canada.

${ }^{4}$ New World Laboratories, Laval, Canada.

${ }^{5}$ KITE Toronto Rehabilitation Institute, University Health Network, Toronto, Canada.
} 
on soft hydrogel substrates that mimic the elasticity of muscle (12 kilopascals) self-renew and contribute extensively to muscle regeneration when transplanted into mice. Conversely, muscle stem cells plated on rigid plastic dishes did not have similar regenerative potential. ${ }^{7}$ Gradients of stiffness in the ECM can also direct migration; this process is known as durotaxis. Durotaxis plays a role in development, fibrosis, and cancer-however, the exact mechanisms used to guide cells along the gradient of stiffness, similar to galvanotaxis, are unknown. Biomechanical properties of the microenvironment can change depending on the neurological diseases (e.g., brain tumors, multiple sclerosis, Alzheimer's disease), ${ }^{8}$ therefore studying galvanotactic response on different substrates is critical.

Herein, we examine the galvanotactic response of clinically relevant human directly reprogrammed NPCs (drNPCs). ${ }^{9}$ In a $2 \mathrm{D}$ in vitro model, we show that drNPCs undergo rapid and directed migration in the presence of a $250 \mathrm{mV} / \mathrm{mm} \mathrm{EF}$, and that the direction of migration (anodal vs. cathodal) can be modulated by the substrate stiffness. We have found that the migration of drNPCs under an applied EF is independent of secreted factors from neighboring cells and extracellular $\mathrm{pH}$. drNPCs migrate anodally on softer substrates, including cell monolayers, and migrate cathodally on stiffer substrates.

\section{Materials and Methods}

\section{Cell culture}

drNPCs were generated from mature human bone marrow cells as previously described. ${ }^{9}$ The drNPCs were cultured in low oxygen conditions in basal medium (NeuroCult ${ }^{\mathrm{TM}} \mathrm{NS}-\mathrm{A}$ Basal Medium Human - Catalog \#05750) containing 10\% proliferation supplement (NeuroCult Proliferation Supplement Human - Catalog \#05753), fibroblast growth factor-2 (FGF-2; $30 \mathrm{ng} / \mathrm{mL}$, Peprotech), epidermal growth factor (EGF, 20ng/mL; Peprotech), and heparin $(100 \mu \mathrm{g} / \mathrm{mL}$; Scientific Protein Laboratories), termed EFH. drNPCs were cultured as free-floating colonies (neurospheres) for 3-7 days on an ultra-low attachment 24-well plate (CLS3473 SigmaAldrich). Individual neurospheres ( $\sim 100 \mu$ m diameter) were collected and plated onto galvanotaxis chambers for 17-20 $\mathrm{h}$ at $37^{\circ} \mathrm{C}$ to allow cell adhesion onto the substrate.

\section{Galvanotaxis chamber}

The galvanotaxis chamber has been previously described. ${ }^{10}$ Chambers were UV sterilized, coated with poly-Llysine (P4707; Sigma-Aldrich), and incubated for $2 \mathrm{~h}$ at room temperature (RT). The central trough was rinsed with autoclaved water; coated with Corning Matrigel Basement Membrane Matrix (4\% v/v; BD Biosciences, Catalog \#354234), Corning Matrigel Growth Factor Reduced (GFR) Basement Membrane Matrix (4\% v/v; BD Biosciences, Catalog \# 354230), or fibronectin $(50 \mu \mathrm{g} / \mathrm{mL}$; Sigma-Aldrich, Catalog \#F1141); and incubated for $1 \mathrm{~h}$ at $37^{\circ} \mathrm{C}$. The central trough was rinsed $3 \times$ with the drNPC supplemented basal medium, and it was covered with $300 \mu \mathrm{L}$ of the media. Neurospheres (4-6) were plated onto the central trough, and the chambers were incubated for $17-20 \mathrm{~h}$ at $37^{\circ} \mathrm{C}$.

For co-culture experiments, drNPCs were plated on matrigel or fibronectin at a density of 100,000 cells/cham- ber and differentiated in $10 \%$ fetal bovine serum (FBS) for 10-15 days. One day before the galvanotaxis assay, DiI (Vybrant; Molecular Probes) labeled drNPCs were plated (10,000 cells/chamber) onto the differentiated monolayer. For drNPC labeling, cells were labeled with $5 \mu \mathrm{L}$ of DiI at a density of $1,000,000$ cells $/ \mathrm{mL}$ for $20 \mathrm{~min}$ at $37^{\circ} \mathrm{C}$, and they were consequently washed $3 \times$ before plating onto the cell monolayer. Co-culture chambers were placed in the incubator overnight at $37^{\circ} \mathrm{C}$ before performing the galvanotaxis assay.

\section{Galvanotaxis assay}

As previously demonstrated, ${ }^{10}$ a glass slide was used to create a roof over the central trough, creating a central chamber. A media reservoir was created on either side of the chamber and sealed with vacuum grease to allow for the electric current flow through the chamber. Silver/silver chloride electrodes were placed in the media of the two side dishes found on either side of the chamber, and the electrodes were connected to an external constant voltage power supply to establish a direct-current EF (DCEF). Cell migration analysis was recorded by using time-lapse imaging microscopy. Images were taken at a frequency of one image per minute for the duration of $3 \mathrm{~h}$ at a magnification of $10 \times$. For experiments involving DiI, images were taken at a frequency of one image every $10 \mathrm{~min}$.

\section{Quantification of cell migration}

Cell migration analysis was carried out by using the automated tracking module of Zeiss Axiovision software. Cells analyzed were at least one cell body away from the nearest neighbor to avoid cells overlapping during migration. Cells closer than one cell body were manually tracked by using the Zeiss Axiovision tracking module. For each experimental group, $\geq 44$ cells were analyzed from $\geq 3$ independent experiments.

Three kinematic parameters were analyzed:

(1) Displacement: the displacement of cells in the direction of the positive or negative $\mathrm{X}$-axis (parallel to the DCEF vector).

(2) Speed: The speed was calculated at two temporal resolutions. Unless otherwise stated, the speed was calculated by taking the total displacement between the initial and final positions of the cells and dividing that by the total experimental time. A higher temporal resolution was used as a point of comparison, and the speed was calculated by averaging the speed in 20min intervals across the $3 \mathrm{~h}$. Each speed was calculated by dividing the displacement by the time elapsed (20 min).

(3) Directedness: The directedness was taken to be positive in the DCEF direction (positive $x$-axis), and negative in the direction opposite to the DCEF (negative $x$-axis). Dividing the displacement along the DCEF vector by the total $(\mathrm{x}, \mathrm{y})$-displacement between the initial and final positions of the cells yields the directedness. Anodal migration was considered to have a negative directedness value, whereas cathodal directedness was positive. 
For individual cell migration path plots, each cell path was plotted starting with a $(0,0)$ origin. ${ }^{11}$ drNPCs were considered to migrate toward the cathode or anode if their displacement toward a terminal was greater than the average of all cell displacements in the absence of an EF plus the standard error means for the respective substrates. ${ }^{11}$ The cell was considered undirected if it did not meet these conditions.

\section{Immunocytochemistry}

drNPCs were fixed with $4 \%$ paraformaldehyde on the central trough of the chambers for $20 \mathrm{~min}$ at RT, followed by $3 \times 5$ min washes with phosphate-buffered saline (PBS). Cells were permeabilized by using $0.3 \%$ Triton X-100 detergent (Sigma-Aldrich) for $20 \mathrm{~min}$ at RT, followed by three washes (with the exception of the anti-fibronectin stain where cells were washed directly and not permeabilized). Cells were exposed to 5\% normal goat serum (Jackson Immunoresearch Laboratories) and $1 \%$ bovine serum albumin in PBS for $1 \mathrm{~h}$ at RT. Cells were incubated with primary antibodies overnight at $4^{\circ} \mathrm{C}$ [rabbit polyclonal anti-SOX2 (1:200; Abcam); rabbit polyclonal anti-fibronectin (1:400; Sigma-Aldrich)]. Chambers were washed $3 \times$ with PBS and then incubated with secondary antibodies: goat anti-rabbit conjugated with Alexafluor 488 (1:400, fibronectin; Invitrogen-Gibco) and goat-anti-rabbit conjugated with Alexafluor 568 (1:400, SOX2; Invitrogen-Gibco) for $1 \mathrm{~h}$ at RT, followed by $3 \times \mathrm{PBS}$ washes. Chambers were incubated with Hoechst $(1: 1000$; Thermofisher) for $30 \mathrm{~min}$ followed by $3 \times$ PBS. Samples were mounted with mounting media (Vector Laboratories), coverslipped, and finally stored at $-20^{\circ} \mathrm{C}$.

\section{Conditioned media}

Conditioned media were collected from drNPCs plated on matrigel substrate (six-well plate, Catalog\#140675; Thermofisher) for $24 \mathrm{~h}$. The media were extracted from the wells and added for galvanotaxis of drNPCs plated on a fibronectin substrate. Controls used media from drNPCs plated on a fibronectin substrate.

\section{$\mathrm{pH}$ measurements}

drNPCs were plated on matrigel or fibronectin substrates as described for $17-20 \mathrm{~h}$ at $37^{\circ} \mathrm{C}$. Media were collected from the central trough of the chambers, and $\mathrm{pH}$ was measured by using UltraBasic benchtop pH Meter (Denver Instrument).

\section{Atomic force microscopy}

Atomic force microscopy (AFM) was performed in PeakForce Tapping mode by using a BioScope Resolve AFM (Bruker Nano Surfaces). PeakForce QNM-Live Cell (PFQNM-LC) probes (Bruker AFM Probes) (tip length $17 \mu \mathrm{m}$, tip radius $65 \mathrm{~nm}$, opening angle $15^{\circ}$ ) were used for imaging. Spring constants of the probes were precalibrated by the manufacturer. ${ }^{12}$

AFM was performed on the surfaces of the central trough of chambers. Imaging was conducted in media with sample heating set to $37^{\circ} \mathrm{C}$. Images were acquired at a scan rate of $0.3 \mathrm{~Hz}$ and $256 \times 256$ pixels with a PeakForce Tapping frequency of $1 \mathrm{kHz}$ and an amplitude of $300 \mathrm{~nm}$. The images were analyzed by using Gwyddion $2.41 .{ }^{13}$ For cell monolayers, images corresponding to the stiffness data channel were not processed before being exported in the .xyz file format, which lists the stiffness value for each pixel, for further analysis. For fibronectin and matrigel substrates, comparison of the height and stiffness data channels included patches corresponding to low stiffness $(<1 \mathrm{MPa})$, surrounded by areas that were lower in height and corresponded to high stiffness ( $>1 \mathrm{MPa})$. To confirm that these raised patches with relatively lower stiffness comprised fibronectin or matrigel, substrates were made from fluorescently-tagged fibronectin and matrigel and combined AFM-fluorescence microscopy was used to verify that the patches corresponded to the fluorescently-tagged substrates, thereby confirming that the drNPCs were plated on coated surfaces. For images comprising uniform height and stiffness values, the stiffness values were exported in the .xyz file format for further analysis. For images with patches of fibronectin or matrigel, a mask was applied to the height images and transferred to the stiffness images to isolate the stiffness values corresponding to the coating material (i.e., fibronectin or matrigel) versus the surrounding area (presumed to be the glass substrate that was coated with either fibronectin or matrigel). Stiffness values corresponding to the masked areas were exported in the .xyz file format for further analysis.

The stiffness values in the .xyz file format were used to calculate the mean, standard deviation, and standard error mean of stiffness for each 2D stiffness map. The stiffness measurements for each 2D stiffness map were plotted in a histogram format to ensure a normal or skewed normal distribution of stiffness values. Due to the large number of stiffness measurements per image $(65,536$ pixels per $256 \times 256$ image), the analysis was performed by using a custom script for Python 3.6.4 with NumPy, Matplotlib, Pandas, and Seaborn packages.

\section{Statistical analysis}

Values are presented as average of total number of cells across all experiments \pm standard error of the mean. Group means were compared by using a Kolmogorov-Smirnov test and an unpaired $t$-test (for comparing two sets of data), along with a one-way ANOVA with a Bonferroni post hoc test and the Kruskal-Wallis non-parametric test followed by Dunn's correction for multiple pairwise comparisons (for comparing three or more sets of data). Kolmogorov-Smirnov and Kruskal-Wallis tests were selected when not all data sets sufficiently fit a normal distribution. Statistical significance was set at $p<0.05$.

\section{Results}

\section{Undifferentiated drNPCs migrate under an applied EF in a substrate-dependent manner}

Human NPCs were plated onto galvanotaxis chambers coated with physiologically relevant substrates in the presence of growth factors. Once cells had adhered to the substrate $\left(17-20 \mathrm{~h}\right.$ at $\left.37^{\circ} \mathrm{C}\right)$, an EF was applied and the cell migration parameters (directedness and speed) were analyzed (Fig. 1a, b). Previous studies have demonstrated that humanderived pluripotent stem cells are electrosensitive cells and undergo galvanotaxis on matrige ${ }^{14}$; hence, in a first series of experiments, we varied the voltage $(0-500 \mathrm{mV} / \mathrm{mm})$ on matrigel to determine the effects of field strength on drNPC 
a

Plated undifferentiated drNPC spheres

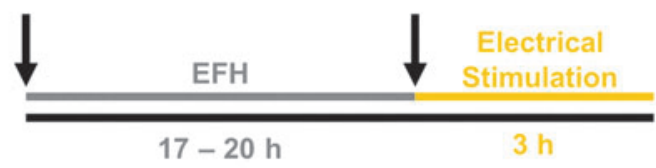

$17-20 \mathrm{~h}$

C
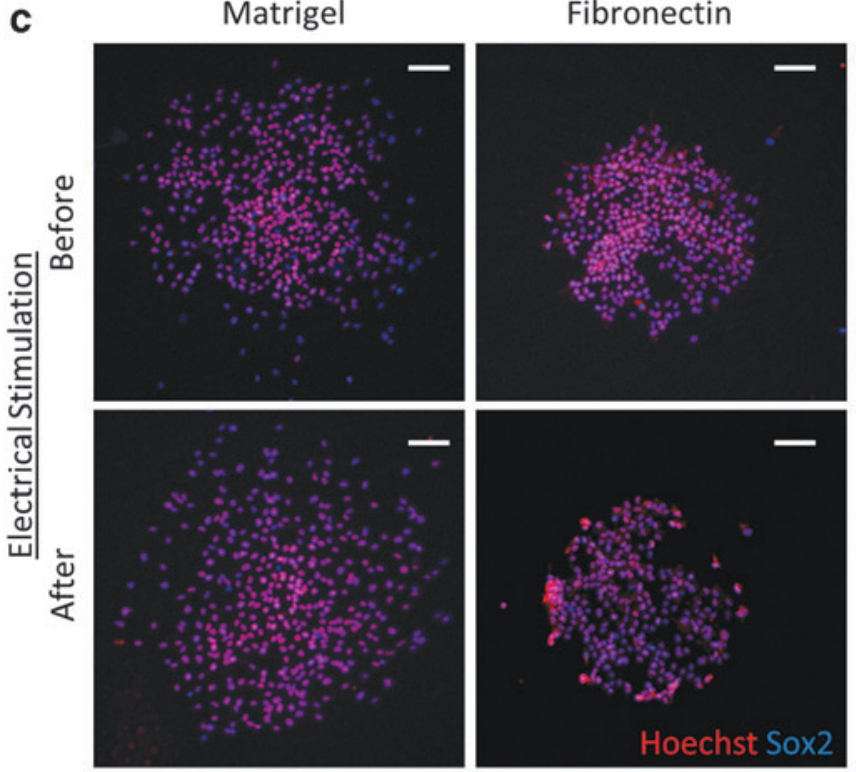

d
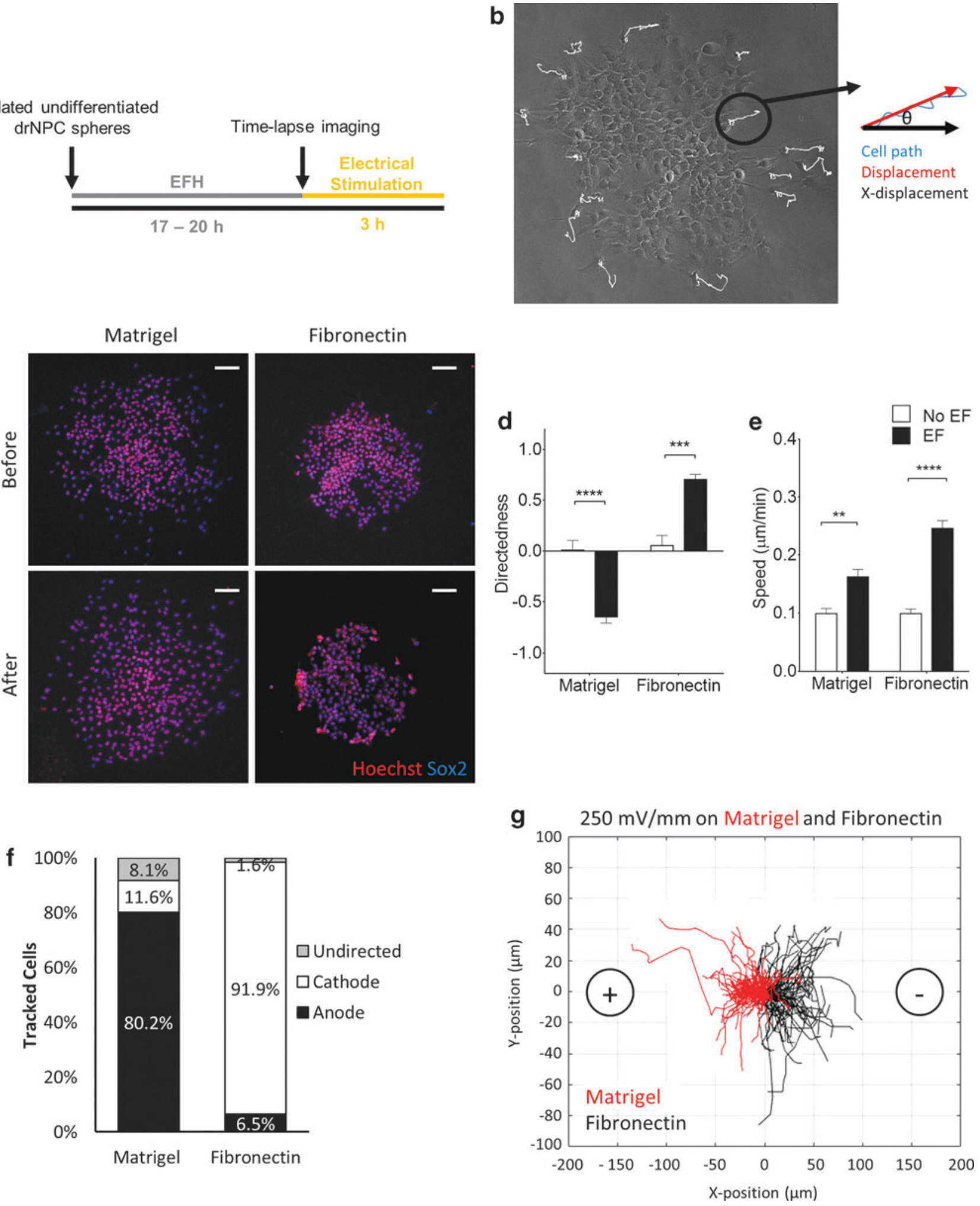

FIG. 1. drNPCs undergo directed migration under an applied EF. (a) Experimental timeline of the galvanotaxis assay. (b) Kinematic parameters analyzed during cell migration. (c) Images of SOX2-positive drNPCs plated on galvanotaxis chambers coated with matrigel and fibronectin before and after electrical stimulation. Scale bars $=100 \mu \mathrm{m}$. Average drNPC migration directedness (d) and speed (e) in the presence and absence of an EF on matrigel and fibronectin substrates. Each bar represents $\geq 45$ cells from $\geq 3$ independent experiments. (f) The percentage of cells that migrated anodally, cathodally, or undirected under an applied EF on the matrigel and fibronectin substrates. (g) Representative plots of individual cell migration paths for drNPCs plated on matrigel $(n=86$ cells) and fibronectin ( $n=62$ cells) under an applied EF. Each line represents one cell with the starting points of the migration path transposed to 0,0. Data are represented as means \pm SEM. $* * p<0.005, * * * p<0.0005 * * * * p<0.0001$. EF, electric field; drNPC, directly reprogrammed neural precursor cell. 
migration. We found that a field strength of $250 \mathrm{mV} / \mathrm{mm}$ was optimal in terms of directedness (anodally) of drNPC migration, compared with no EF controls (Supplementary Fig. S1 and Supplementary Movie S1). Accordingly, this stimulation paradigm was used for all subsequent experiments.

We performed immunocytochemical analysis of the cells both before and after EF application on matrigel and fibronectin by using transcription factor SOX2, a marker of undifferentiated drNPCs, to determine whether the $250 \mathrm{mV} / \mathrm{mm}$ EF induced drNPC differentiation. ${ }^{15}$ Consistent with murine NPC studies, we found that $>97 \%$ of the drNPCs expressed the undifferentiated cell marker both before and after stimulation (Fig. 1c).

Next, the speed and directedness were assessed. drNPCs migrated with increased speed (analyzed at the 3-h interval and at 20-min intervals) and increased directedness in the presence of the EF, compared with no EF conditions (Fig. 1d, e and Supplementary Fig. S2) on both matrices. Strikingly, the cells migrated in opposite directions depending on the substrate, moving anodally (toward the positive terminal) on the matrigel substrate and cathodally on fibronectin (Fig. 1d).

The percentage of migrating cells moving toward the anode, cathode, or undirected was analyzed. The majority of tracked cells migrated in a directed fashion on both matrices $(91.9 \%$ [fibronectin] and $80.2 \%$ [matrigel]) (Fig. 1f). The prominent difference in the direction of migration on matrigel versus fibronectin (anodal vs. cathodal, respectively) (Fig. 1g) prompted us to explore the extracellular factors that contributed to the EF-induced migration parameters.

\section{Growth factor composition and $\mathrm{pH}$ of substrate do not alter the directedness of drNPC migration}

We next asked whether factors present in matrigel could account for the different (anodal) direction of migration. Matrigel is extracted from mouse sarcoma and contains a variety of growth factors, including Platelet-derived growth factor (PDGF), Insulin-like growth factor 1 (IGF-1), and transforming growth factor beta 1 (TGF- $\beta$ ) (Table 1). ${ }^{16} \mathrm{We}$ examined the galvanotactic response of drNPCs on standard matrigel and GFR matrigel with reduced levels of PDGF, IGF-1, and TGF- $\beta$. Irrespective of the growth factor reduction, drNPCs migrated anodally, and with the same degree of directedness (matrigel: $-0.88 \pm 0.04$, GFR matrigel: $-0.75 \pm 0.06$ ) (Fig. 2a). Hence, these growth factors were not sufficient to regulate the directedness of drNPC migration.

In a next series of experiments, we asked whether factors found in conditioned media from drNPCs cultures on matrigel would be sufficient to change the galvanotactic response of drNPCs plated on fibronectin (i.e., drNPCs would

Table 1. Growth Factor Composition of Matrigel

\begin{tabular}{lrr}
\hline Growth factor & Matrigel & GFR matrigel \\
\hline NGF & $<0.2 \mathrm{ng} / \mathrm{mL}$ & $<0.2 \mathrm{ng} / \mathrm{mL}$ \\
PDGF & $5-48 \mathrm{pg} / \mathrm{mL}$ & $<5 \mathrm{pg} / \mathrm{mL}$ \\
IGF-1 & $11-24 \mathrm{ng} / \mathrm{mL}$ & $5 \mathrm{ng} / \mathrm{mL}$ \\
TGF- $\beta$ & $1.7-4.7 \mathrm{ng} / \mathrm{mL}$ & $1.7 \mathrm{ng} / \mathrm{mL}$ \\
\hline
\end{tabular}

GFR, growth factor reduced; PDGF, platelet-derived growth factor; IGF-1, insulin-like growth factor 1 ; TGF- $\beta$, transforming growth factor beta 1 . change their direction of migration from cathodal [fibronectin] to anodal, in the presence of factors released from matrigel-exposed drNPCs). drNPCs were plated on fibronectin and exposed to an $\mathrm{EF}$ in conditioned media derived from drNPCs on matrigel. We found that drNPCs continued to migrate cathodally, the direction in which they would normally migrate on fibronectin, despite the presence of conditioned media (Fig. 2b). Together, these findings support the hypothesis that the substrate dictates the direction of migration.

Previous studies have revealed that extracellular $\mathrm{pH}$ is sufficient to change the direction of migration of human keratinocytes in an applied EF. ${ }^{17}$ Based on this observation, we asked whether extracellular $\mathrm{pH}$ may play a role in the anodal versus cathodal migration observed on matrigel and fibronectin substrates, respectively. The $\mathrm{pH}$ of media at the time of EF application (17-20 h of plating) was similar between matrigel $(7.88 \pm 0.03)$ and fibronectin $(7.91 \pm 0.06)$ substrates (Fig. 2c), suggesting that $\mathrm{pH}$ may not be a factor accounting for the anodal versus cathodal migration for drNPCs in an applied EF.

\section{Undifferentiated drNPCs migrate anodally on a monolayer of differentiated neural cells}

To better mimic the physiological conditions in vivo, we plated drNPCs onto a cell monolayer comprising neurons, astrocytes, and oligodendrocytes from differentiated drNPCS, as previously described..$^{9}$ First, we examined the migratory behavior of differentiated drNPCs in the presence of an EF. Briefly, drNPCs were differentiated on a galvanotaxis chamber coated with matrigel for 10-15 days, in the presence of $10 \%$ FBS, and an EF was applied across the chamber. Consistent with previous findings using differentiated mouse-derived NPCs, differentiated human cells did not migrate in a directed manner $(0.05 \pm 0.09)$, and they traveled with a reduced speed $(0.07 \pm 0.01 \mu \mathrm{m} / \mathrm{min}) \mathrm{com}$ pared with undifferentiated drNPCs with a directedness of $-0.71 \pm 0.10$ and a speed of $0.27 \pm 0.06 \mu \mathrm{m} / \mathrm{min}$ (Fig. 3a).

Next, undifferentiated drNPCs were plated onto the differentiated drNPC monolayer and their directedness was examined. The cell monolayers were differentiated on either matrigel or fibronectin. Undifferentiated drNPCs were fluorescently-labeled with the cell membrane marker DiI before plating on the cell monolayers to enable visualization and cell tracking in the galvanotactic assay (Fig. 3b, c). We found that DiI-labeled, undifferentiated drNPCs migrated anodally on the monolayer, irrespective of the underlying substrate (matrigel or fibronectin), and similar to what was observed when drNPCs were plated directly on matrigel (matrigel only: Directedness $=-0.65 \pm 0.05$; cell monolayer on matrigel: Directedness $=-0.67 \pm 0.05$; and cell monolayer on fibronectin: Directedness $=-0.83 \pm 0.04$, Fig. $3 \mathrm{~d}$, e).

We next sought to determine whether substrate binding, or the negative charge of fibronectin, was sufficient to direct the anodal versus cathodal migration. We plated undifferentiated drNPCs on a fibronectin-coated cell monolayer, confirming the presence of fibronectin on the monolayer by using fluorescent labeling (Fig. 3b, f). We predicted that if substrate binding or the charge of fibronectin was playing a role in directedness, then the drNPCs would migrate as if they were on fibronectin alone (i.e., they would migrate cathodally). We 
a

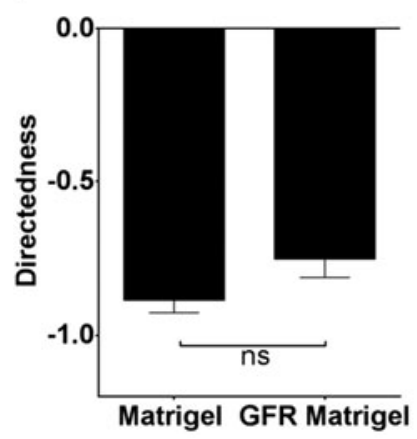

b

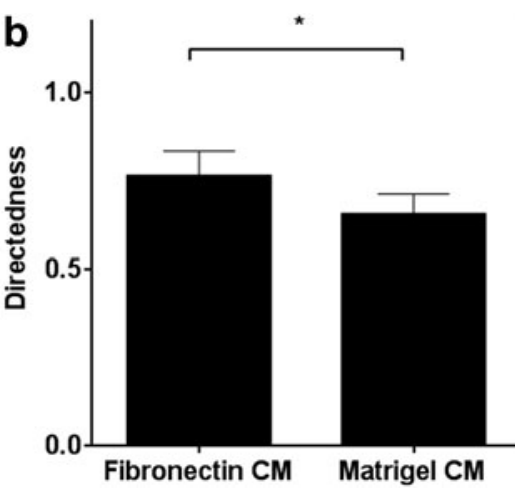

C

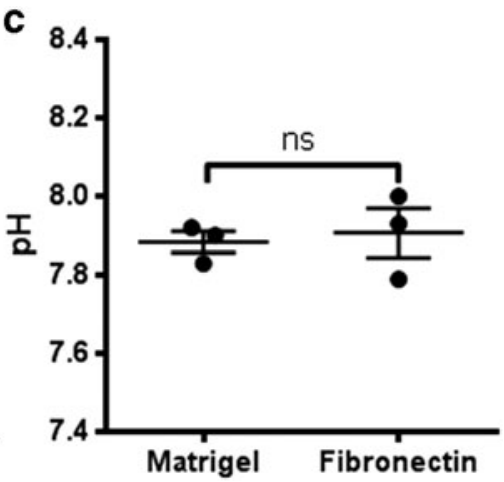

FIG. 2. Growth factor composition and $\mathrm{pH}$ of substrate do not play a role in the direction of drNPC migration under applied EFs. (a) drNPCs directedness on matrigel and GFR matrigel in an EF ( $n \geq 45$ cells from $\geq 3$ independent experiments for each condition). (b) drNPC directedness on fibronectin in the presence of conditioned media derived from cultures of cells plated on fibronectin or matrigel, in an applied EF ( $n \geq 45$ cells from $\geq 3$ independent experiments for each condition). (c) Extracellular $\mathrm{pH}$ after incubating drNPCs on different substrates for $17-20 \mathrm{~h}$ measured on matrigel $(n=3)$ and fibronectin $(n=3)$. CM = conditioned media. Data are represented as means \pm SEM. $* p<0.05$. CM, conditioned media; GFR, growth factor reduced; ns, non-significant.

found that drNPCs plated on fibronectin-coated cell monolayers migrated anodally (Fig. 3d, e), identical to what was observed on the cell monolayers alone and matrigel alone. These findings suggest that downstream signaling pathways activated by fibronectin ligand binding on drNPCs, and fibronectin's negative charge, are not responsible for the directedness of migration.

\section{Substrate stiffness plays a role in the direction of migration in an applied EF}

Substrate stiffness has been shown to play a role in stem cell behavior, including stable focal adhesion formation, ${ }^{6}$ stem cell differentiation, ${ }^{18,19}$ migration, ${ }^{20,21}$ and proliferation. ${ }^{22}$ We asked whether substrate stiffness variation could account for the anodal versus cathodal migration observed by drNPCs in the presence of an EF. We used AFM to investigate the stiffness of the substrates (matrigel, fibronectin, cell monolayer, and fibronectin-coated cell monolayer). AFM images acquired in Peakforce Tapping mode enabled simultaneous topology imaging and 2D-mapping of mechanical properties, including stiffness. Stiffness maps were acquired that demonstrated that fibronectin-coated chambers had a significantly greater stiffness than the cell monolayer (Fig. 4) $(p<0.05)$. We also found large variation of substrate stiffness in fibronectin compared with matrigel or the cell monolayer (Supplementary Fig. S3). The stiffness of the cell monolayer and fibronectin-coated cell monolayer was not significantly different from matrigel $(p=0.58$ and $p=0.98$ respectively) (Fig. 4). These findings suggest that the mechanical properties of the substrate play a role in determining the directionality of drNPC migration under an applied EF.

\section{Discussion}

Understanding the migratory behavior of NPCs under applied EFs is important for potential clinical applications to treat CNS injuries. The aim of this study was to determine the galvanotactic response of human drNPCs in a physiologically relevant EF strength. We have shown that human drNPCs are electrosensitive cells that undergo directed migration that is substrate-dependent and correlated with substrate stiffness. Our findings support the hypothesis that the mechanical properties of the substrate play a vital role in the direction of migration under an applied EF.

Previous studies have demonstrated the importance of the microenvironment in galvanotaxis. Babona-Pilipos et al. demonstrated that the small molecule Erlotinib, which inhibits the EGF receptor activity, significantly reduces the velocity of NPC migration in an applied EF. ${ }^{2}$ Further, ECM molecules can modulate human keratinocyte galvanotaxis affecting their velocity. ${ }^{23}$ Previous studies have demonstrated that the charge of the substrate can regulate the direction of xenopus spinal neurite outgrowths. ${ }^{24}$ Our studies demonstrate, for the first time, that charge alone is not sufficient to change the direction of cell migration and that a change in the direction of migration (anodal vs. cathodal) can be achieved by plating the same cell type on different substrates with varied stiffness. ${ }^{25}{ }^{25}$ Indeed, adult murine NPCs undergo rapid and cathodally directed migration irrespective of the substrate onto which NPCs are plated (unpublished observation) ${ }^{2}$ and spatial configuration (2D vs. 3D). ${ }^{26}$

Interestingly, our initial studies with matrigel and fibronectin suggest that the cause for differential migration is not soluble $(\mathrm{pH}$ remained similar between substrates whereas conditioned media from matrigel on fibronectin and reduced growth factors for matrigel did not reverse the migration). Further, neither fibronectin's negative charge nor its integrinbinding proteins were causing the difference in migratory direction as drNPCs plated on fibronectin-coated cell monolayers migrated anodally, which is opposite to their direction of migration on fibronectin alone.

Our data are consistent with the conclusion that substrate stiffness regulates the direction of migration. The cell monolayer was significantly stiffer than fibronectin, the only substrate on which the cells migrated cathodally. Although we found no significant difference between matrigel and fibronectin substrate stiffness, the direction of migration was the same on all of the less-stiff substrates. We observed a large variation in the fibronectin stiffness analyses and we 
a

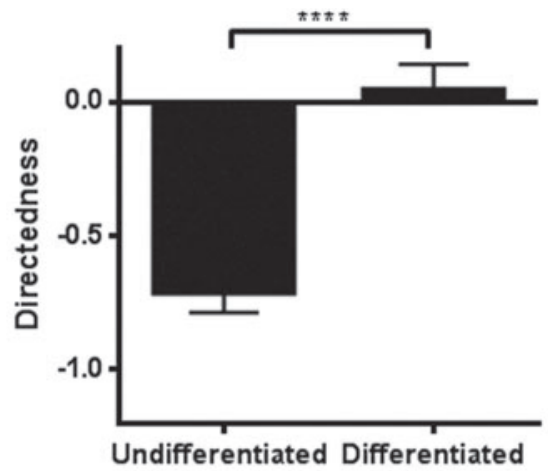

C

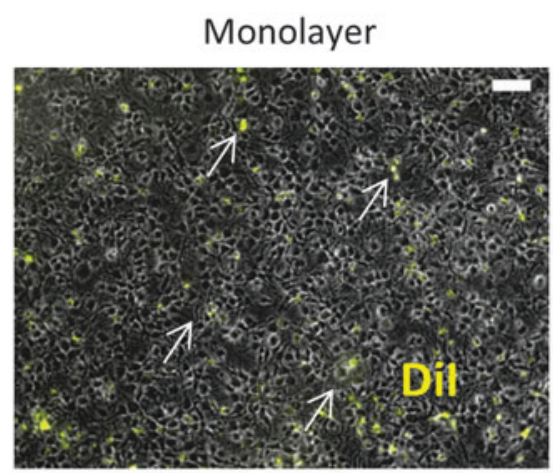

e

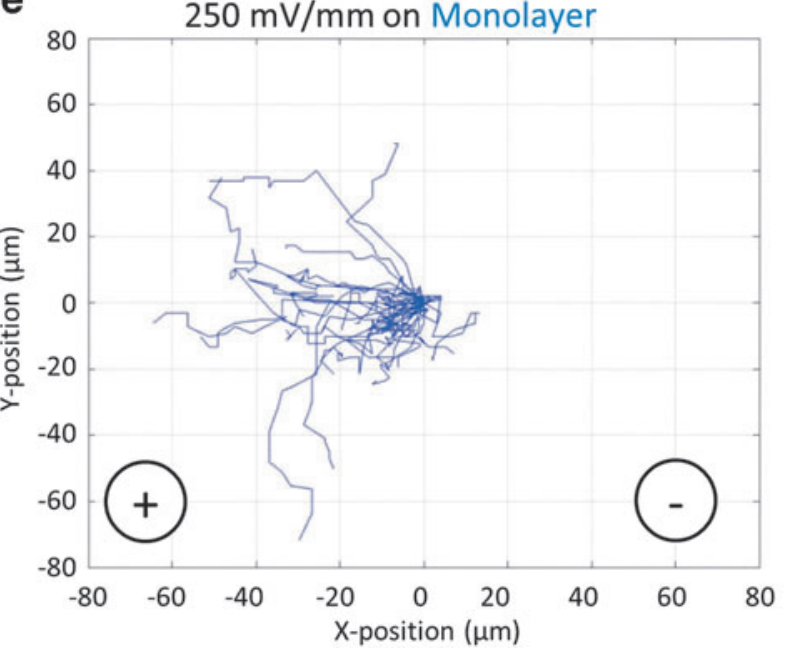

b

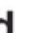

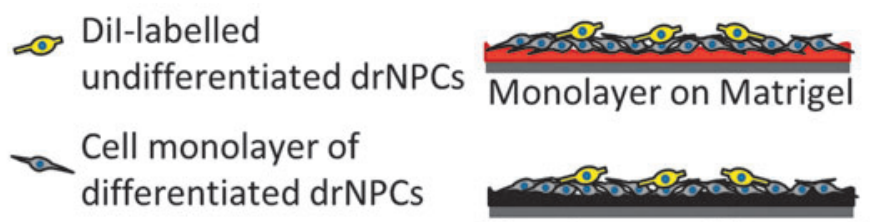

- Matrigel

Monolayer on Fibronectin

- Fibronectin

- Glass slides

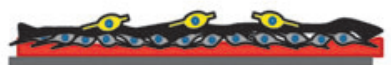

Fibronectin-Coated Monolayer

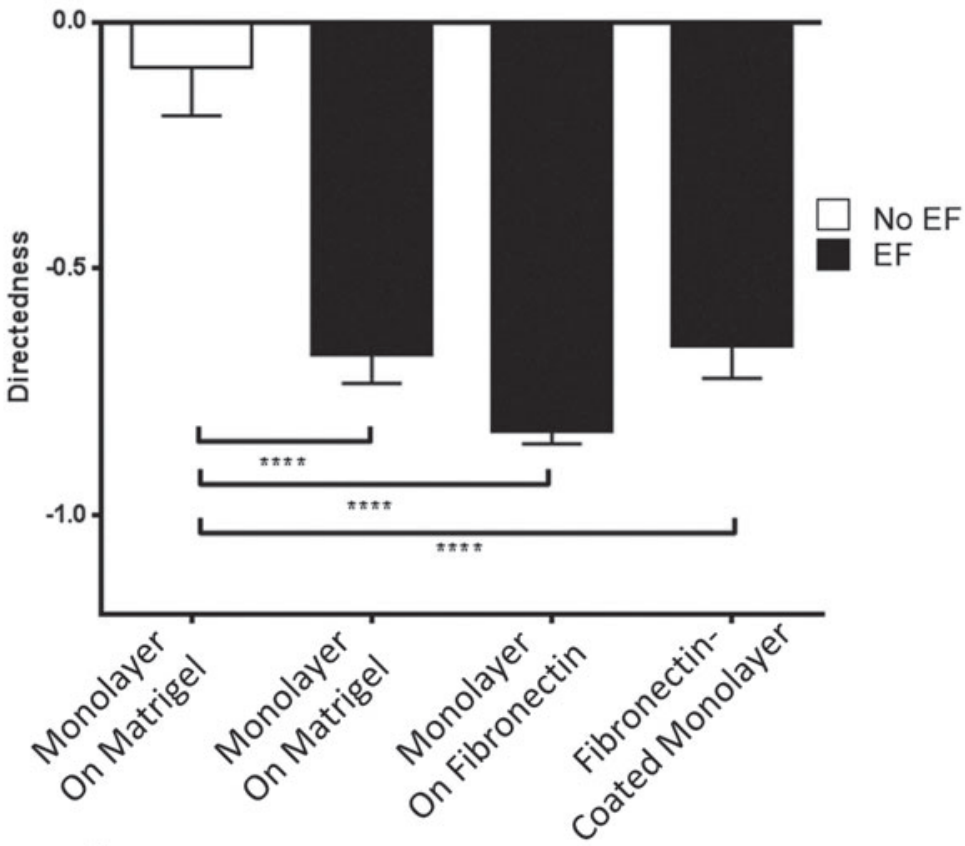

f

Monolayer, not coated with Fibronectin

Fibronectin-Coated

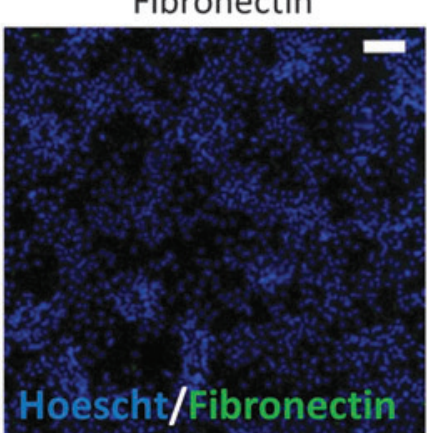

Monolayer

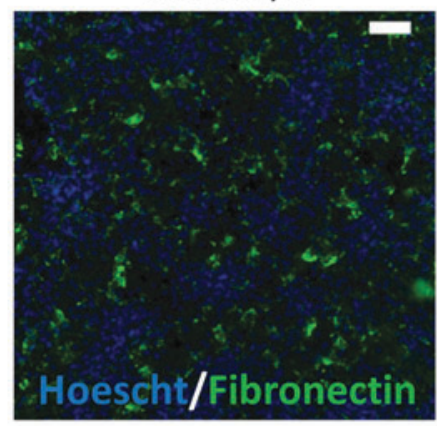

FIG. 3. The use of differentiated drNPC monolayer as a substrate elicits anodal migration of undifferentiated drNPCs under an applied EF. (a) Directed migration of differentiated, but not undifferentiated, drNPCs in the presence of an EF. (b) Experimental set-up of combinations of substrates with cell monolayers. (c) Fluorescent image of the cell monolayer plated with DiI-labeled, undifferentiated drNPCs (arrows). Scale bar $=100 \mu \mathrm{m}$. (d) Directedness of DiI-labeled drNPCs in the presence or absence of an EF when plated on a cell monolayer (on matrigel or fibronectin) or plated on the surface of a fibronectin-coated cell monolayer. Each bar represents $\geq 45$ cells from $\geq 3$ independent experiments for each group. Means were compared by using Kruskal-Wallis test followed by Dunn's correction for multiple pairwise comparisons. Data are represented as means \pm SEM. (e) Representative plots of individual cell migration paths for drNPCs plated on a cell monolayer ( $n=45$ cells) under an applied EF. Each line represents one cell. (f) Hoescht-labeled nuclei (blue) of a cell monolayer, with and without a fibronectin coated surface. Scale bar $=100 \mathrm{um}$. $* * * * p<0.0001$. 


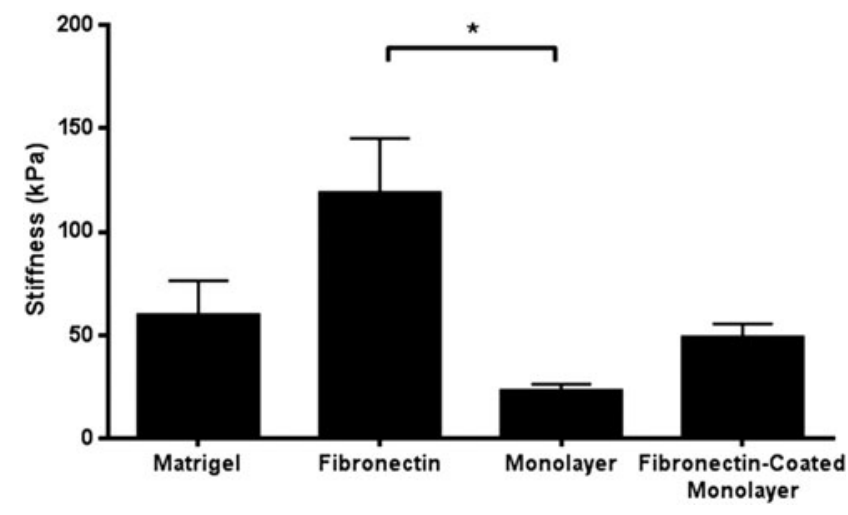

FIG. 4. Average stiffness measurements of substrates. The average stiffness and standard error mean from AFM measurements of complete coatings of matrigel $(n=5$ samples), fibronectin ( $n=6$ samples), cell monolayer $(n=4$ samples), and cell monolayer coated with fibronectin $(n=4$ samples). ${ }^{*} p<0.05$. AFM, atomic force microscopy.

cannot rule out the fact that the range of stiffness found only on fibronectin could be important for cathodal migration.

Within the CNS, mechanical properties of the tissue have been found to vary according to region as well as pathological conditions. ${ }^{27}$ After injury to the CNS, a glial scar is formed and found to be softer-less stiff-compared with healthy CNS tissue. ${ }^{28}$ Biological factors such as age and sex have also been found to affect tissue biomechanics such as stiffness. ${ }^{8}$ Therefore, knowledge on the effect of mechanical properties of the substrate on which NPCs migrate is vital for designing potential neurorepair strategies that employ EFs for cell stimulation.

There are numerous proposed mechanisms for sensing and transducing EFs into cellular migration. It is well established that ion channels play a critical role in sensing and transducing EFs. ${ }^{29}$ In addition, a reduction in extracellular $\mathrm{pH}$ has been found to induce a change in directionality from cathodal to anodal galvanotaxis of human keratinocytes through inhibition of G-protein-coupled receptor/purinergic pathway signaling. ${ }^{17}$ Although not through a change of $\mathrm{pH}$ in our setup a proposed mechanism is that the inhibition of the G-protein-coupled receptor at lower substrate stiffness (i.e., matrigel and cell monolayer substrates) may induce a change in directionality from cathodal to anodal migration. Next steps involve studying the activation of specific pathways, such as the pathways downstream of G-protein-coupled receptors, in response to substrate stiffness to determine how matrix stiffness may alter directedness of human NPC migration.

\section{Conclusion}

Herein, we have explored the galvanotaxis of physiologically relevant drNPCs, specifically studying the effect of ECM, secreted factors of neighboring cells, as well as extracellular $\mathrm{pH}$ on the directedness of drNPC migration. Our results indicate that the directedness of drNPC galvanotactic migration is, indeed, substrate-dependent, and altering the stiffness of the substrate can change the directionality of drNPC migration. To fully utilize the therapeutic potential of NPCs in CNS neurorepair strategies, it is critical to under- stand the migratory behavior of human NPCs to enhance the initial step of NPC mobilization from their subependymal zone niche and migration to the lesion site.

\section{Author Confirmation Statement}

U.A designed experiments; acquired, analyzed, and interpreted data; and wrote the article. S.N.I. analyzed and interpreted the data and wrote the article. L.P. designed AFM experiments; acquired, analyzed, and interpreted AFM data. M.R.P. and C.Y. interpreted data and provided financial support. C.M.M. conceived and designed experiments, analyzed and interpreted data, provided financial support, and wrote the article. J.-E.A. interpreted the data and provided financial support.

All co-authors have reviewed and approved of the article before submission. The article has been submitted solely to this journal and is not published, in press, or submitted elsewhere. There is a publicly available preprint on SSRN.

\section{Acknowledgment}

The authors would like to thank Vianney Delplace and Nancy Liu for helpful discussions.

\section{Author Disclosure Statement}

J.E.A. is a shareholder of New World Laboratories, Inc.

\section{Funding Information}

This research was funded by the Canadian Institutes of Health Research and New World Laboratories, Inc.

\section{Supplementary Material}

Supplementary Figure S1

Supplementary Figure S2

Supplementary Figure S3

Supplementary Movie S1

\section{References}

1. Kahle MP, Bix GJ. Neuronal Restoration Following Ischemic Stroke. Neurorehabil Neural Repair 2013;27:469478.

2. Babona-Pilipos R, Droujinine IA, Popovic MR, et al. Adult subependymal neural precursors, but not differentiated cells, undergo rapid cathodal migration in the presence of direct current electric fields. PLoS One 2011;6:e23808.

3. Ferrier J, Ross SM, Kanehisa J, et al. Osteoclasts and osteoblasts migrate in opposite directions in response to a constant electrical field. J Cell Physiol 1986;129:283-288.

4. Wang E, Zhao M, Forrester J V., et al. Bi-directional migration of lens epithelial cells in a physiological electrical field. Exp Eye Res 2003;76:29-37.

5. Wen J, Vincent L, Fuhrmann A, et al. Interplay of matrix stiffness and protein tethering in stem cell differentiation. Nat Mater 2014;13:979-987.

6. Trappmann B, Gautrot J, Connelly J. Extracellular-matrix tethering regulates stem-cell fate. Nat Mater 2012;11:642649.

7. Gilbert P, Havenstrite K, Magnusson K, et al. Substrate elasticity regulates skeletal muscle stem cell self-renewal in culture. Science (80-) 2010;329:1078-1081. 
8. Arani A, Murphy MC, Glaser KJ, et al. Measuring the effects of aging and sex on regional brain stiffness with MR elastography in healthy older adults. Neuroimage 2015; 111:59-64.

9. Ahlfors JE, Azimi A, El-Ayoubi R, et al. Examining the fundamental biology of a novel population of directly reprogrammed human neural precursor cells. Stem Cell Res Ther 2019;10:1-17.

10. Babona-Pilipos R, Popovic MR, Morshead CM. A galvanotaxis assay for analysis of neural precursor cell migration kinetics in an externally applied direct current electric field. J Vis Exp 2012;88:4193.

11. Iwasa SN, Popovic MR, Morshead CM. Skin-derived precursor cells undergo substrate-dependent galvanotaxis that can be modified by neighbouring cells. Stem Cell Res 2018; 31:95-101.

12. Schillers H, Medalsy I, Hu S, et al. PeakForce Tapping resolves individual microvilli on living cells. J Mol Recognit 2016;29:95-101.

13. Nečas D, Klapetek P. Gwyddion: An open-source software for SPM data analysis. Open Phys 2012;10:181-188.

14. Zhang J, Calafiore M, Zeng Q, et al. Electrically guiding migration of human induced pluripotent stem cells. Stem Cell Rev Rep 2011;7:987-996.

15. Ellis P, Fagan BM, Magness ST, et al. SOX2, a persistent marker for multipotential neural stem cells derived from embryonic stem cells, the embryo or the adult. Dev Neurosci 2004;26:148-165.

16. Corning ${ }^{\circledR}$ Matrigel ${ }^{\circledR}$ Matrix Frequently Asked Questions. www.corning.com/catalog/cls/documents/faqs/CLS-DL-CC026.pdf. Last accessed January 24, 2020.

17. Saltukoglu D, Grünewald J, Strohmeyer N, et al. Spontaneous and electric field-controlled front-rear polarization of human keratinocytes. Mol Biol Cell 2015;26: 4373-4386.

18. Oh S, An D, Kim T, et al. Wide-range stiffness gradient PVA/HA hydrogel to investigate stem cell differentiation behavior. Acta Biomater 2016;35:26-31.

19. Engler A, Sen S, Sweeney H, et al. Matrix elasticity directs stem cell lineage specification. Cell 2006;126:677-689.

20. Trichet L, Le Digabel J, Hawkins R, et al. Evidence of a large-scale mechanosensing mechanism for cellular adap- tation to substrate stiffness. Proc Natl Acad Sci U S A 2012;109:6933-6938.

21. Ng M, Besser A, Danuser G. Substrate stiffness regulates cadherin-dependent collective migration through myosin-II contractility. J Cell Biol 2012;199:545-563.

22. Leipzig N, Shoichet M. The effect of substrate stiffness on adult neural stem cell behavior. Biomaterials 2009;30: 6867-6878.

23. Sheridan DM, Isseroff RR, Nuccitelli R. Imposition of a physiologic DC electric field alters the migratory response of human keratinocytes on extracellular matrix molecules. J Invest Dermatol 1996;106:642-646.

24. Rajnicek AM, Robinson KR, McCaig CD. The direction of neurite growth in a DC electric field depends on the substratum. Mol Biol Cell 1998;9:226A.

25. Babona-Pilipos R, Liu N, Pritchard-Oh A, et al. Calcium influx differentially regulates migration velocity and directedness in response to electric field application. Exp Cell Res 2018;368:202-214.

26. Meng X, Arocena M, Penninger J, et al. PI3K mediated electrotaxis of embryonic and adult neural progenitor cells in the presence of growth factors. Exp Neurol 2011;227: 210-217.

27. Holtzmann K, Gautier HOB, Christ AF, et al. Brain tissue stiffness is a sensitive marker for acidosis. J Neurosci Methods 2016;271:50-54.

28. Moeendarbary E, Weber I, Sheridan G, et al. The soft mechanical signature of glial scars in the central nervous system. Nat Commun 2017;8:14787.

29. Nakajima K, Zhu K, Sun YH, et al. KCNJ15/Kir4.2 couples with polyamines to sense weak extracellular electric fields in galvanotaxis. Nat Commun 2015;6:8532.

Address correspondence to:

Cindi M. Morshead, PhD

Department of Surgery

University of Toronto

160 College Street, Rm 1006

Toronto M5S 3E2

Canada

Email: cindi.morshead@utoronto.ca 